\title{
Transrectal ultrasound-guided seminal vesicle catheterization with continuous antibiotic infusion for the treatment of refractory hematospermia
}

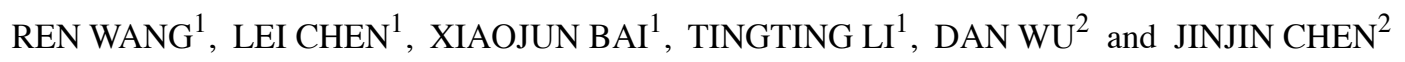 \\ ${ }^{1}$ Department of Ultrasound in Medicine, Shanghai Jiao Tong University Affiliated Sixth People's Hospital, \\ Shanghai Institute of Ultrasound in Medicine, Shanghai 200233; ${ }^{2}$ Children Health Care Center, \\ Shanghai Children's Hospital Affiliated to Shanghai Jiao Tong University, Shanghai 200060, P.R. China
}

Received May 21, 2020; Accepted October 23, 2020

DOI: $10.3892 /$ etm.2020.9464

\begin{abstract}
The aim of the present study was to describe transrectal ultrasound (TRUS)-guided seminal vesicle catheterizations with continuous antibiotic infusion in patients with persistent hematospermia. A retrospective record review of 45 patients with refractory hematospermia treated with TRUS-guided seminal vesicle catheterization between 2010 and 2017 was performed. Seminal vesicle puncture and catheterization was performed under TRUS guidance for all patients. Antibiotic irrigation was used to rinse the seminal vesicle until the outflow fluid was clear. The trocar sleeve was left in situ and fixed on the skin of the perineum at the end of the procedure. All patients underwent a 24-h continuous infusion of antibiotic solution through the catheter. The patients were followed up to 3 years for the presence of hematospermia. The duration of refractory hematospermia was between 6 months and 9 years. A total of 14 patients exhibited consecutive hematospermia, while the remaining patients exhibited intermittent episodes. On TRUS, 15 cases of ejaculatory duct cyst, 7 cases of ejaculatory duct expansion, 3 cases of ejaculatory duct stones, 6 cases of seminal vesicle expansion, 8 cases of seminal vesicle stones and 5 cases of seminal vesicle wall or ejaculation wall calcification were diagnosed. A total of 41 patients completed the scheduled treatment plan; however, the catheter was dissociated on the $3 \mathrm{rd}$ or 4 th day of catheterization in 4 patients.
\end{abstract}

Correspondence to: Dr Ren Wang, Department of Ultrasound in Medicine, Shanghai Jiao Tong University Affiliated Sixth People's Hospital, Shanghai Institute of Ultrasound in Medicine, 600 Yishan Road, Shanghai 200233, P.R. China

E-mail: renwang7474@163.com

Dr Jinjin Chen, Children Health Care Center, Shanghai Children's Hospital Affiliated to Shanghai Jiao Tong University, 355 Luding Road, Shanghai 200060, P.R. China

E-mail:jjvoo@163.com

Key words: hematospermia, transrectal ultrasound, catheterization, treatment
After a 1-3 year follow-up, hematospermia was not observed in 42 patients $(93.33 \%)$ with recurrence in the remaining 3 patients. In conclusion, TRUS-guided seminal vesicle catheterization with continuous antibiotic infusion appeared to be a safe and effective method for the treatment of hematospermia.

\section{Introduction}

Hematospermia is a common symptom in urology, which is defined as visible blood in the ejaculate $(1,2)$. It is commonly seen in patients $<40$ years old with a worldwide prevalence of one case per 5,000 urological patients (1). The exact prevalence of the condition is difficult to determine as only a few men examine their ejaculate and even less report to a clinician for a consultation (3). Hematospermia can be an isolated condition or can occur with other symptoms such as hematuria, dysuria and/or scrotal pain $(3,4)$. It may occur only once in a life-time of an individual or can be periodic and chronic (3-5).

Hematospermia is usually benign and a self-healing condition that is less frequently associated with a underlying pathological process (5). The etiology of hematospermia is often not known in $>70 \%$ cases; however, with advances in diagnostic technologies, the causes of hematospermia are more readily recognized (1). Persistent or recurrent hematospermia is often caused by non-specific inflammation or infection of the lower urinary tract, especially seminal vesiculitis and prostatitis $(3,4)$. In some cases, pathologies such as benign urethral tumor or seminal vesicle malignancy may also present as hematospermia $(5,6)$.

A thorough clinical work-up including the patients' medical history, sexual history, blood biochemistry, physical examination and examination of the ejaculate is recommended for assessing all patients presenting with the condition (6). In the majority of cases, hematospermia resolves without any treatment and in patients without any underlying cause of risk factors, only reassurance is sufficient to alleviate the patients anxiety $(1,5,6)$. In the remaining patients, management of hematospermia depends on the underlying cause. In cases of infections, empirical administration of antibiotics often treat the condition successfully (4). Surgical treatment may be required in cases of pathological abnormalities like cysts, vascular anomalies or calcifications (6). While the majority of patients 
with hematospermia are cured in a short period of time, a small number of patients relapse with persistent symptoms, and the management of these patients can be challenging (3).

Recent advances in the field of minimally invasive surgery has led to development of trans urethral seminal vesiculoscopy for the management of recurrent hematospermia (7). However, limitations of this technique include the degree of difficulty involved with the procedure and the surgical experience required (8). A simpler technique involving image-guided puncture of seminal vesicles and irrigation with antibiotic solution has received limited attention in literature $(1,5)$. The current study presents the results of the use of transrectal ultrasound (TRUS)-guided seminal vesicle catheterizations with continuous antibiotic infusion for the treatment of patients with persistent hematospermia.

\section{Patients and methods}

Patients. A retrospective record review of 45 male patients with refractory hematospermia treated with TRUS-guided seminal vesicle catheterization at Shanghai Jiao Tong University Affiliated Sixth People's Hospital between January 2010 and December 2017 was performed. The Ethics Committee of Shanghai Sixth People's Hospital (Shanghai, China) approved the present study (approval no. 2020-KY-010) and written informed consent was obtained from all patients. The age range of the 45 patients was between 25-67 years. All patients had undergone non-invasive treatments for hematospermia including oral antibiotics, prostate massage and physical therapy among others. Only the patients whose symptoms of hematospermia were not controlled or recurred $>3$ times in 2 years were included in the present study. Preoperative hematological tests, ultrasound and MRI were performed to exclude patients with tumors in the prostate, seminal vesicles, bladder, testis and epididymis. Patients with coagulopathy caused by systemic diseases were also excluded. Before treatment, digital rectal examination was performed to obtain prostate secretions, which were subjected to bacterial culture and drug sensitivity tests. All patients with a positive bacterial culture underwent a full course of oral antibiotic therapy based on the drug sensitivity tests.

Procedure. The patients were advised to not ejaculate for $>1$ week prior to catheterization to ensure that the seminal vesicle was sufficiently sized. Yum MyLab 90 and MyLab Twice color Doppler ultrasound (Esaote) equipped with a transrectal biplane probe were used. 6F drainage trocars (Bard; Becton, Dickinson and Company or Bioteque Corporation) were used for catheterization.

With patients in lithotomy position, the transrectal biplane probe was used for ultrasound examination to observe the morphology of the prostate, seminal vesicle, ejaculatory duct and internal echo. The probe also aided in the examination of ejaculatory duct stone formation and to exclude any tumors of the reproductive system. When the seminal vesicle images were clearly displayed on the longitudinal cross sections of the sonogram, the seminal vesicles were noted on the patient's body. Thorough perineal disinfection was performed in all patients. Lidocaine hydrochloride $(5 \mathrm{ml})$ was then infiltrated at the catheter puncture site and around the prostate capsule. The puncture
Table I. Baseline characteristics of the study patients.

\begin{tabular}{ll}
\hline Characteristic & \multicolumn{1}{c}{ Data } \\
\hline Number of patients, $\mathrm{n}$ & \multicolumn{1}{c}{45} \\
Age, years (mean \pm SD) & $40.87 \pm 7.24$ \\
Age range (years) & $25-67$ \\
Hematospermia, $\mathrm{n}(\%)$ & \\
Consecutive & $14(31.11)$ \\
Intermittent & $31(68.88)$ \\
Symptoms, $\mathrm{n}(\%)$ & \\
Perineal discomfort & $29(64.44)$ \\
Ejaculation pain & $17(37.77)$ \\
Sexual dysfunction & $12(26.66)$ \\
Hematuria & $3(6.66)$ \\
Duration of hematospermia, months & $33.11 \pm 19.68$ \\
(mean \pm SD) & \\
Range (months) & $6-108$ \\
Pathology on preoperative TRUS & \\
examination, $n(\%)$ & \\
Single pathology & $17(37.78)$ \\
Multiple pathology & $12(26.67)$ \\
No pathology detected & $16(35.55)$ \\
\hline
\end{tabular}

TRUS, transrectal ultrasound; SD, standard deviation.

point was identified $\sim 1 \mathrm{~cm}$ away and 30-45 degrees from the anus. Under ultrasound guidance, the $6 \mathrm{~F}$ trocar was inserted into the seminal vesicles (Figs. 1 and 2). Following withdrawal of the needle, the seminal vesicle fluid was obtained for examination. Irrigation with antibiotic solution was repeatedly performed to rinse the seminal vesicle until the outflow fluid was clear. The trocar sleeve was left in situ and fixed on the skin of the perineum with sutures. All patients underwent a 24 -h continuous infusion with an antibiotic solution via the catheter after the procedure. The antibiotic solution constituted of 120,000 units of gentamicin per side in $500 \mathrm{ml}$ normal saline, which was irrigated at a rate of 4-6 drops per minute for a slow and continuous infusion. Gentamicin was selected in the present study, as it has been indicated to be effective against staphylococci and gram-negative bacilli (9). These microorganisms were consistently observed in the bacterial cultures of the patient population with seminal vesiculitis of the current study. Broad-spectrum antibiotics, such as cephalosporins, were not used due to their cost and the high percentage of drug resistance in the patients of the present study. For patients with a positive culture of prostate secretion, the antibiotic solution was selected according to the results of the drug allergy test. The treatment lasted for 7 days and the catheter was removed at the end of the treatment.

Statistical analysis. Continuous variables were presented as mean \pm SD while categorical data was presented as number (\%). Pre- and post-treatment outcomes were compared using Fisher's exact test. Statistical analysis was performed using Stata version 11 (StataCorp LP). 




Figure 1. Trocar insertion into the seminal vesicle through the prostate. The horizontal arrow indicates the seminal vesicle, and the vertical arrow indicates the trocar.

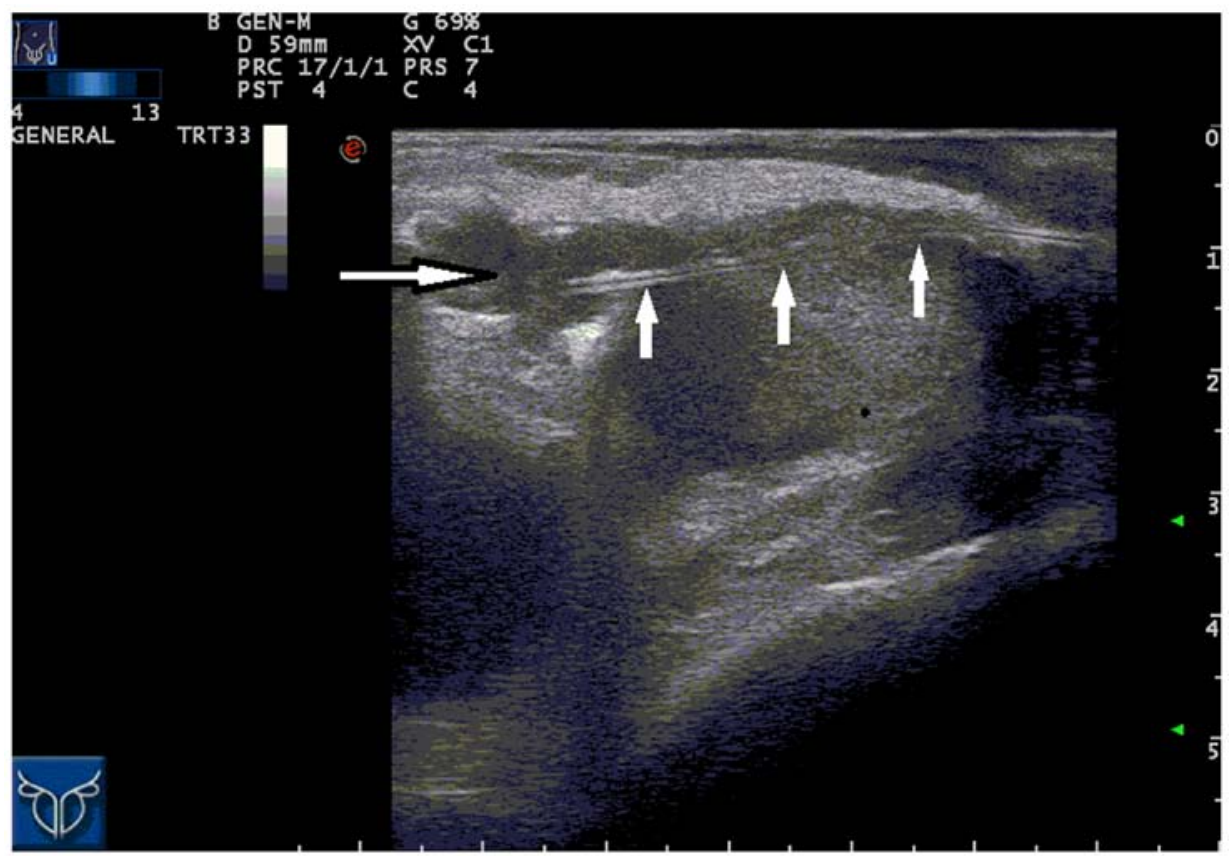

Figure 2. Indwelling trocar following successful puncture of the seminal vesicle. The horizontal arrow indicates the seminal vesicle, and the vertical arrow indicates the trocar.

\section{Results}

Characteristics of patients. Details of patients are presented in Table I. The duration of the refractory hematospermia was between 6 months and 9 years. A total of 14 patients exhibited consecutive hematospermia, while the remaining 31 patients exhibited intermittent episodes of hematospermia. The most common additional symptoms were perineal discomfort (29 patients), ejaculation pain (17 patients), sexual dysfunction (12 patients) and hematuria (3 patients). No patient reported a history of interventional procedure for any seminal vesicle or prostate pathology.

On preoperative examination with TRUS, no pathology was detected in 16 patients, while the remaining patients exhibited single or multiple pathologies (Table I). A total of 15 cases of ejaculatory duct cyst, 7 cases of ejaculatory duct expansion, 3 cases of ejaculatory duct stones, 6 cases of seminal vesicle expansion, 8 cases of seminal vesicle stones and 5 cases of seminal vesicle wall or ejaculation wall calcification were observed prior to treatment (Table II). 
Table II. Post-treatment outcomes.

\begin{tabular}{lcccc}
\hline Pathology & Pre-treatment, $\mathrm{n}$ & Post-treatment, $\mathrm{n}$ & Total healed, $\mathrm{n}(\%)$ & P-value \\
\hline Ejaculatory duct cyst & 15 & 8 & $7(46.6)$ & 0.14 \\
Ejaculatory duct expansion & 7 & 2 & $5(71.4)$ & 0.15 \\
Ejaculatory duct stones & 3 & 0 & $3(100.0)$ & 0.24 \\
Seminal vesicle expansion & 6 & 0 & $6(100.0)$ & 0.02 \\
Seminal vesicle stones & 8 & 3 & $5(62.5)$ & 0.19 \\
Seminal vesicle wall or ejaculation wallcalcification & 5 & 3 & $2(40.0)$ & 0.71
\end{tabular}

Preoperative prostatic fluid examination of $32(71.11 \%)$ patients demonstrated higher white blood cell levels (>10/high power field) compared with normal levels, while $8(17.78 \%)$ patients exhibited a positive bacterial culture with 3 cases being positive for Escherichia coli, 2 for Proteus 2 for Staphylococcus aureus and 1 for Staphylococcus epidermidis. In the seminal vesicle fluid analysis, $11(24.44 \%)$ patients exhibited blood-containing fluid in bilateral seminal vesicles and $23(51.11 \%)$ patients exhibited blood-containing fluid in unilateral seminal vesicles, which was mostly brown and viscous. Fluid from the seminal vesicle of remaining $11(24.44 \%)$ patients could not be withdrawn, apart from a small amount of light reddish liquid after rinsing. No tumor cells were observed in the seminal vesicle fluids, and 2 cases exhibited a positive bacterial culture of pathogenic Escherichia coli on semen analysis.

Treatment. All 45 patients were treated with continuous antibiotic irrigation via the seminal vesicle, which were catheterized successfully in the first attempt. The procedure was well tolerated by all patients. No patient reported severe pain resulting in discontinuation of the treatment. All patients exhibited different degrees of hematuria following catheter insertion, which was healed after 1-3 days. A total of 41 patients completed the scheduled treatment plan; however, the catheter was dissociated on the 3rd or 4th day of catheterization in 4 patients. Extravasation of antibiotics leading to scrotal edema was observed in 4 patients, and as a result an early catheter removal was performed.

Follow-up. TRUS examinations were conducted 3 months after the treatment. Complete healing was observed in all cases of ejaculatory duct stones and seminal vesicle expansion (Table II). The healing percentage of the other pathologies ranged from 40 to $71.4 \%$. Only healing of the seminal vesicle expansion was statistically significant $(\mathrm{P}<0.05)$. The total follow-up ranged between 1 and 3 years. No occurrence of hematospermia was observed in 42 patients $(93.33 \%)$. A total of 3 patients exhibited recurrence of hematospermia at 6,12 and 18 months after treatment, 2 of whom had not completed the scheduled treatment plan due to catheter dissociation.

\section{Discussion}

The causes of hematospermia include inflammation, tumor formation, congenital malformations and iatrogenic injuries on seminal vesicles, prostate, testis, epididymis, bladder and urethra (7). More commonly, inflammation of the seminal vesicles and prostate is commonly associated with the condition while iatrogenic factors such as prostate biopsy, radiotherapy for prostate cancer are also frequently observed $(4,5)$. The majority of patients with hematospermia can heal on their own within a few weeks. Certain patients exhibit a recurrent blood presence in the semen that results in persistent or refractory hematospermia (6). These symptoms can give rise to anxiety in patients, leading to erectile dysfunction and infertility in certain more severe cases (10).

Despite the varying etiologies of hematospermia, the common pathophysiology of the disease involves obstruction of the seminal vesicles owing to inflammation, and management of the inflammation usually resolves the condition $(11,12)$. Conventional treatment includes systemic medication and topical treatment, such as prostate seminal vesicle massage, hot water bath and physiotherapy, to improve blood circulation in local tissues in order to promote the absorption and discharge of inflammatory substances (11).

For patients with refractory hematospermia, conventional treatments often yield poor efficacy (7). This is determined by the anatomy and physiological characteristics of the seminal vesicle and prostate that consists of a pair of highly folded and coiled tubular glands. The base of the mucosal folds in the lumen of the seminal vesicle is surrounded by numerous tortuous diverticula $(4,10)$. When the seminal vesicle is inflamed, the drainage is not smooth or even obstructed due to the structural characteristics of the vesicle (11). In addition, the blood supply of the seminal vesicle comes from the small branch of the inferior bladder artery, therefore blood circulation is poor. The effects of the barrier of fibrosis around the prostate capsule limits the ability of antibiotics to access the prostate epithelium (1,3,7). In addition, antibiotics are usually water soluble and exhibit acidity that lowers their dissociation constant, rendering them able to bind easily to proteins (9). These properties additionally limit the antibiotics' accessibility to the infected area and indicate that systemic medications do not exhibit a bactericidal effect in the prostate and seminal vesicles $(6,9)$. Therefore, the prostate and seminal vesicle bacteria are difficult to eliminate, and the lesions are not easy to heal (12). In order to solve this problem, certain researchers have used TRUS to guide the treatment of refractory hematospermia by a single injection of antibiotics, and achieved a curative effect (12-14). Topical medication can significantly increase the concentration of the drug in the lesion, so that a bactericidal effect can be 
achieved (14). However, since the injected antibiotics are usually quickly discharged into the bladder via the ejaculation tube, the antibiotics can only remain in the seminal vesicle for relatively short time. Therefore, the function of the antibiotics cannot be fully exerted, and the recurrence rate of hematospermia remains high $(12,14)$. In the present study, the 24-h continuous antibiotic infusion via the seminal vesicle catheter with the guidance of TRUS was indicated to aid in maintaining an effective antibacterial concentration in the seminal vesicle and prostate for sufficiently longer time. It can be hypothesized that the potential presence of posterior urethritis and epididymitis can also be treated concomitantly with the infiltration and dispersion of the antibiotic. This also aids to prevent the recurrence of prostatitis and seminal vesiculitis caused by adjacent lesions after treatment.

Previous studies on hematospermia have indicated that recurrent hematospermia was associated with chronic inflammation of the prostate and seminal vesicles $(13,14)$. Chronic inflammation and its secondary alterations cause obstruction, stone formation and stenosis (15-17). Moreover, the inflammation-induced formation of stones, obstruction and stenosis can worsen the inflammation rendering it more difficult to heal, as a vicious circle $(15,18,19)$. Preoperative examination of the present group of patients revealed that secondary alterations, such as ejaculatory cysts and seminal vesicle stones among others, exhibit higher occurrence rates, which was consistent with previous research findings.

In the present study, the seminal vesicle was placed in a 24-h continuous antibiotic infusion for the treatment of refractory hematospermia. In addition to the antibacterial effect of the drug itself, it is important to continuously supply the drug with a certain pressure to aid the drainage of the seminal vesicle content, which relieves the obstruction or imperfection of the semen drainage channel and promotes the excretion of infectious substances and fine stones (14).

The treatment was indicated to be effective on both bacterial and non-bacterial inflammation. Out of 45 patients of the present study, hematospermia disappeared in 42 patients (93.33\%). The efficacy of the present treatment was revealed to be higher compared with the method of antibiotic supply using a single seminal vesicle puncture (14). In parallel, secondary lesions from inflammation, such as obstructions and stones, were also alleviated to a certain degree. Moreover, reduction of the likelihood of recurrence was achieved, as only 3 patients exhibited recurrence in the current study. This may be attributed to the dissociation of the catheter in 2 patients, which led to incomplete treatment. The obstruction of the seminal vesicle may not have been relieved, or the infection of the vesicles may not have been eliminated due to incomplete treatment or presence of resistant microorganisms. Large stones in the seminal vesicles may also not be removed with catheterization and may harbor microbes leading to recurrence.

The clinical value of seminal vesicle ultrasound has gradually been recognized with its application in diseases, such as refractory hematospermia and obstruction, in recent years (20). The main goal of seminal vesicle ultrasound is to clarify the patency of the ejaculatory duct, remove the cause of obstruction or malaise and improve the excretion of the seminal vesicle for therapeutic purposes (21). Although the ultrasound-guided treatments are effective in relieving the seminal vesicle obstruction, they exhibit certain limitations (20). On the one hand, finding the ejaculatory duct opening during surgery and entering into the seminal pouch is difficult and the possibility of failure in the operation is high; on the other hand, surgery may also lead to complications, such as rectal injury, external urinary sphincter injury, retrograde ejaculation, urine reflux and secondary infection (21-26).

In the present study, it was revealed that TRUS-guided seminal vesicle catheterization was an effective and easy approach for reaching the seminal vesicle, with good success rates and a high reproducibility, since the operation can be repeated when necessary. In the present study, all 45 patients were successfully catheterized at the first attempt without any complications, except for hematuria after operation, which was indicated to be safe. One of the most critical aspects of seminal vesicle catheterization is to determine whether the puncture needle has entered the seminal vesicle. Patients with chronic seminal vesiculitis often demonstrate poor sound transmission inside the seminal vesicle (14). In the present study, the structure of the seminal vesicle in the ultrasound image could not be distinguished from that of the prostate based on the images alone, as it could be determined whether the catheter has entered the seminal vesicle. During the procedure, although there was a slight puncture when the needle passed via the prostate into the seminal vesicle, the puncture was often not evident and could not be distinguished in all procedures. When it was determined via monitoring of the ultrasonic images that the puncture needle had entered the seminal vesicle, the successful aspiration of the seminal vesicle fluid aided in indicating that the catheter was in place. If the fluid withdrawal was unsuccessful, a small amount of normal saline was injected. If the injection encountered resistance, it indicated that the puncture needle was still in the prostate area, and the needle was required to be inserted deeper. On the other hand, if saline injection was successful, it indicated that that the puncture needle had entered the seminal vesicle. The rapid expansion of the seminal vesicles, which was accompanied by a shining light spot's quick 'rolling' motion, could be observed in the ultrasonic images. Subsequently, the liquid quickly moved into the bladder via the ejaculation tube and the patient exhibited an urge to urinate, which indicated that the puncture needle was at the correct location and the catheter could be fixed at that place. All patients were routinely indwelled with urinary catheters, which aided to promptly drain the irrigation fluid and reduce the patient activity to avoid catheter dissociation.

The present study presents certain limitations. Firstly, hematospermia cases of different etiologies were included in the current study. The actual effect of the present treatment protocol for each etiology of hematospermia should be explored in future studies. Secondly, the sample size of the present study was small with a limited follow-up. There is a need for additional studies with a larger sample size and longer follow-ups to provide in-depth evidence on the efficacy of this technique.

In conclusion, TRUS-guided seminal vesicle catheterization was indicated to be a convenient and safe procedure for the treatment of persistent hematospermia. The continuous infusion of antibiotics on the affected area was revealed to 
be efficient with excellent prospects for clinical application. However, this procedure is invasive for both diagnosis and treatment and should not be used in the case of non-refractory hematospermia.

\section{Acknowledgements}

Not applicable.

\section{Funding}

The present study was funded by Shanghai Key Discipline of Medical Imaging Fund (grant no. 2017 ZZ 02005) and Shanghai Key Clinical Disciplines Fund (grant no. shslczdzk03203).

\section{Availability of data and materials}

The datasets used and/or analyzed during the current study are available from the corresponding author on reasonable request.

\section{Authors' contributions}

RW, LC and JC conceived and designed the study. XB, TL and DW collected the data and performed data analysis. RW and LC wrote the draft of the manuscript. RW and JC edited the manuscript. All authors read and approved the final manuscript.

\section{Ethics approval and consent to participate}

The Ethics Committee of Shanghai Sixth People's Hospital (Shanghai, China) approved the present study (approval no. 2020-KY-010) and written informed consent was obtained from all patients.

\section{Patient consent for publication}

Not applicable.

\section{Competing interests}

The authors declare that they have no competing interests.

\section{References}

1. Mathers MJ, Degener S, Sperling H and Roth S: Hematospermia-a Symptom With Many Possible Causes. Dtsch Arztebl Int 114: 186-191, 2017.

2. Fuse H, Komiya A, Nozaki T and Watanabe A: Hematospermia: Etiology, diagnosis, and treatment. Reprod Med Biol 10: 153-159, 2011.

3. Schiff JD, Bar-Chama N and Schiff HI: Hemospermia: Pathophysiology and Diagnosis - Is Treatment Required? Curr Sex Health Rep 4: 43-48, 2007.

4. Leocádio DE and Stein BS: Hematospermia: Etiological and management considerations. Int Urol Nephrol 41: 77-83, 2009.

5. De Sanctis V, Zargooshi J, Marsciani A, Soliman AT, Elsedfy H, Soliman NA and Di Maio S: For Debate: Should We Worry about Hematospermia in Adolescents and Young Men? A Report of Six Cases and Review of the Literature. Pediatr Endocrinol Rev 14: 281-288, 2017.
6. Khodamoradi K, Kuchakulla M, Narasimman M, Khosravizadeh Z, Ali A, Brackett N, Ibrahim E and Ramasamy R: Laboratory and clinical management of leukocytospermia and hematospermia: A review. Ther Adv Reprod Health 14: 2633494120922511, 2020

7. Liao L-G, Li Y-F, Zhang Y, Li K, Zhu T, Li BJ, Wang Q, Liu XD, Luo Y, Zhou B, et al: Etiology of 305 cases of refractory hematospermia and therapeutic options by emerging endoscopic technology. Sci Rep 9: 5018, 2019.

8. Wang X-S, Li M, Shao G-F, Sun WD, Zhang XL, Xiao ZY, Ma Z, Yuan MZ and Guo LQ: Real-time transrectal ultrasound-guided seminal vesiculoscopy for the treatment of patients with persistent hematospermia: A single-center, prospective, observational study. Asian J Androl 22: 507-512, 2020.

9. Kumar CG, Himabindu M and Jetty A: Microbial biosynthesis and applications of gentamicin: A critical appraisal. Crit Rev Biotechnol 28: 173-212, 2008.

10. Ahmad I and Krishna NS: Hemospermia. J Urol 177: 1613-1618, 2007.

11. Coppens L: Diagnosis and treatment of obstructive seminal vesicle pathology. Acta Urol Belg 65: 11-19, 1997 (In French).

12. Fuse H, Sumiya H, Ishii $H$ and Shimazaki J: Treatment of hemospermia caused by dilated seminal vesicles by direct drug injection guided by ultrasonography. J Urol 140: 991-992, 1988.

13. Zhang K, Li SQ, He ZJ, Jin J, Liu ZJ, Shan GZ and Na YQ: Etiology and management of persistent hematospermia: A pilot study. Zhonghua Nan Ke Xue 9: 118-121, 2003 (In Chinese).

14. Zhang K, Li SQ, He ZJ, Jin J and Na YQ: Long-term efficacy of TRUS-guided transperineal needle aspiration and irrigation on persistent hematospermia. Zhonghua Nan Ke Xue 11: 452-454, 2005 (In Chinese).

15. Xue RZ, Tang ZY, Chen Z and Huang L: Clinical outcomes of transperitoneal laparoscopic unroofing and fenestration under seminal vesiculoscopy for seminal vesicle cysts. Asian J Androl 20: 621-625, 2018.

16. Liu B, Li J, Li P, Zhang J, Song N, Wang Z and Yin C: Transurethral seminal vesiculoscopy in the diagnosis and treatment of intractable seminal vesiculitis. J Int Med Res 42: 236-242, 2014.

17. Hosseinzadeh K, Oto A, Allen BC, Coakley FV, Friedman B, Fulgham PF, Hartman MS, Heller MT, Porter C, Sahni VA, et al; Expert Panel on Urologic Imaging: ACR Appropriateness Criteria ${ }^{\circledR}$ Hematospermia. J Am Coll Radiol 14 (Suppl): S154-S159, 2017

18. Yang SC, Rha KH, Byon SK and Kim JH: Transutricular seminal vesiculoscopy. J Endourol 16: 343-345, 2002.

19. Ozgök Y, Kilciler M, Aydur E, Saglam M, Irkilata HC and Erduran D: Endoscopic seminal vesicle stone removal. Urology 65: 591, 2005.

20. Yagci C, Kupeli S, Tok C, Fitoz S, Baltaci S and Gogus O: Efficacy of transrectal ultrasonography in the evaluation of hematospermia. Clin Imaging 28: 286-290, 2004.

21. Razek AAKA, Elhanbly S and Eldeak A: Transrectal ultrasound in patients with hematospermia. J Ultrasound 13: 28-33, 2010.

22. La Vignera S, Condorelli RA, Vicari E, D'Aagata R, Salemi M and Calogero AE: Hyperviscosity of semen in patients with male accessory gland infection:direct measurement with quantitative viscosimeter. Andrologia 44 (Suppl 1): 556-559, 2012.

23. Paick J, Kim SH and Kim SW: Ejaculatory duct obstruction in infertile men. BJU Int 85: 720-724, 2000.

24. Elia J, Delfino M, Imbrogno N, Capogreco F, Lucarelli M, Rossi T and Mazzilli F: Human semen hyperviscosity: Prevalence, pathogenesis and therapeutic aspects. Asian J Androl 11: 609-615, 2009.

25. Zhang W, Xiao G, Qin S, Reed-Maldonado AB, Xu J, Chen G, Wang $\mathrm{L}$ and Zhou T: An innovative technique of transurethral seminal vesiculoscopy with ultrasonic lithotripter for severe, persistent hematospermia. J Endourol 31: 1277-1282, 2017.

26. Guo S, Xie D, He X, Du C, Zhu L, Deng X and Yang Z: The application of pediatric ureteroscope for seminal vesiculoscopy. Minim Invasive Surg 2015: 946147, 2015.

(i) $(-)$ This work is licensed under a Creative Commons Attribution-NonCommercial-NoDerivatives 4.0 International (CC BY-NC-ND 4.0) License. 